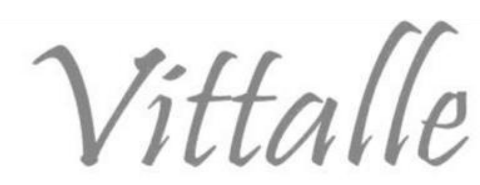

\title{
Neuroartropatia de Charcot secundária à siringomielia com acometimento isolado de ombro: relato de caso
}

\author{
Marcos André Mendes da Silva ${ }^{\mathrm{a}^{*}}$, Claudemir Batista Campos Junior ${ }^{\mathrm{b}}$, Eduardo Menezes \\ Testa $^{\mathrm{b}}$, Gabriel Oberdan Massaru Carvalho Yamao ${ }^{\mathrm{b}}$, Gabriel Rigatti $^{\mathrm{b}}$ \\ ${ }^{a}$ Hospital Universitário Dr. Miguel Riet Corrêa Junior (HU/FURG), Rio Grande, RS, Brasil \\ ${ }^{\mathrm{b}}$ Faculdade de Medicina, Universidade Federal do Rio Grande, Rio Grande, RS, Brasil
}

Histórico do Artigo
Recebido em:
28/01/2020
Aceito em:
08/03/2020
Palavras-chave:
Articulação de
Charcot; artropatia
neuropática;
siringomielia; ombro

\section{Keywords:}

Charcot joint; neuropathic arthropathy; syringomyelia; shoulder

\begin{abstract}
RESUMO
A neuroartropatia de Charcot do ombro é uma doença degenerativa crônica causada pela perda da sensibilidade articular por diferentes etiologias. Quando ocorre secundariamente a siringomielia, encontramos poucos casos descritos na literatura científica. O diagnóstico precoce é de grande valor, uma vez que o quadro clínico se apresenta de forma inespecífica e pode levar a destruição irreversível da articulação. Com o objetivo de demonstrar a excepcionalidade da doença, alertando a comunidade médica sobre a raridade da enfermidade e o diagnóstico diferencial dentre as demais artropatias degenerativas, descrevemos o caso de um paciente do sexo masculino de 56 anos com neuroartropatia isolada no ombro esquerdo secundária a siringomielia, concluindo que o diagnóstico diferencial é crucial para correta conduta terapêutica.
\end{abstract}

Charcot neuroartopathy secondary to siringomielia with isolated shoulder involvement: case report

\section{Introdução}

A neuroartropatia de Charcot, também chamada de artropatia neuropática (NA), é uma doença degenerativa crônica causada pela perda da sensibilidade articular (1). Foi descrita pela primeira vez por Mitchel em 1813 e depois foi nomeada por Jean-Martin Charcot em 1868 (2).

A NA possui diferentes etiologias com predileção por articulações diferentes. O envolvimento de pés/tornozelos é mais comum em diabéticos; o joelho é mais comum na sífilis; e o ombro/cotovelo em pacientes com siringomielia (3). Ainda assim, existem causas mais raras de artropatia de Charcot, entre elas: a lepra, a meningomielocele, o alcoolismo e a insensibilidade congênita à dor (2).

A NA do ombro é um desafio diagnóstico, visto que é confundida com infecções, ruptura tendinosa do manguito rotador, fraturas ou condições patológicas de melhor prognóstico. A causa mais comum de NA no ombro é a siringomielia, uma desordem da medula espinhal com formação e alargamento de uma cavidade central ou siringe. As

\footnotetext{
*Autor correspondente: marcos@ cirurgiadeombrors.com.br (da Silva M.A. M.)
} 
causas mais frequentes de siringomielia são: malformação de Arnold-Chiari tipo I, hidrocefalia comunicante, traumas, tumores medulares, medula presa e sua forma idiopática (4).

Do total de casos de NA, 5-6\% são representados pelo comprometimento do ombro (5, 6). Já entre os pacientes com siringomielia $25 \%$ desenvolvem NA (7). Com isso, o objetivo desse relato é alertar a comunidade médica sobre a raridade da enfermidade e a importância do diagnóstico diferencial da neuroartropatia em relação as demais artropatias degenerativas, visto que o tratamento artroplástico tem altas taxas de falha nestes casos. Além disso, preencher as lacunas da literatura científica buscando estimular maiores investigações da doença e consequentemente tratamentos mais eficazes.

\section{Caso clínico}

Paciente do sexo masculino, 56 anos, residente e natural de São José do Norte, Rio Grande do Sul, pescador há mais de 40 anos, foi encaminhado ao ortopedista devido a queixa de dor progressiva no ombro esquerdo (OE) há 6 meses. Nega trauma recente, dor e inchaço em outras articulações. Apresenta apenas hipertensão arterial sistêmica controlada, nega diabetes, tabagismo e etilismo. No exame físico, aumento de volume ao nível do deltóide (anterior, lateral e posterior) com exacerbação da deformidade a movimentação e sinal de rotura da cabeça longa do musculo bíceps braquial (Figura. 1). Amplitude de movimento com: $100^{\circ}$ de elevação anterior ativa (EAA), $45^{\circ}$ de rotação externa com o braço junto ao corpo (RE1), $90^{\circ}$ de rotação externa com o braço em adução de $90^{\circ}$ (RE2) e rotação interna (RI) mão - nádegas. Testes Irritativos: Neer, Yokum, Hawkins-Kennedy negativos. Manobras para o manguito rotador com Jobe 3+e Patte 3+. Lift off não foi possível de ser realizado por rotação interna insuficiente. Bear Hug e Press Belly test positivos. Já os testes para cabeça longa do músculo bíceps braquial não foram realizados por apresentar sinal de rotura do mesmo. Em relação às manobras deficitárias, constatou-se Drop arm-, Lag sign + e Hornblower +.

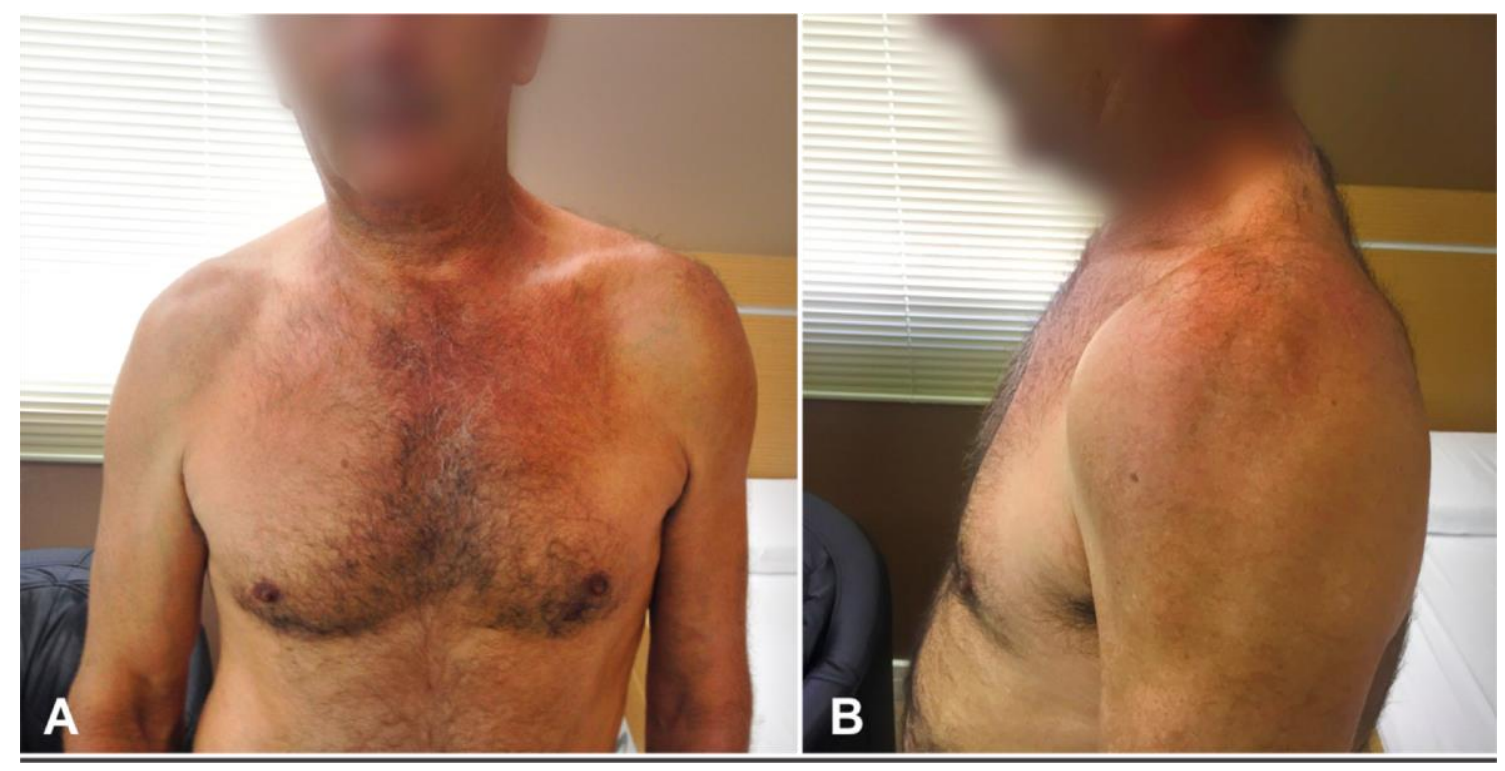

Figura 1 - A. Imagem anteroposterior do paciente demonstrando assimetria do ombro esquerdo em relação ao direito. B. Imagem em perfil evidenciando aumento de volume no ombro esquerdo, especialmente em sua porção posterior. 
A radiografia do ombro esquerdo revelou alterações morfoestruturais por sequela traumática na cabeça umeral, acrômio com sinais de osteoartrose secundária e inúmeros corpos livres osteocondrais periarticulares (Figura 2).

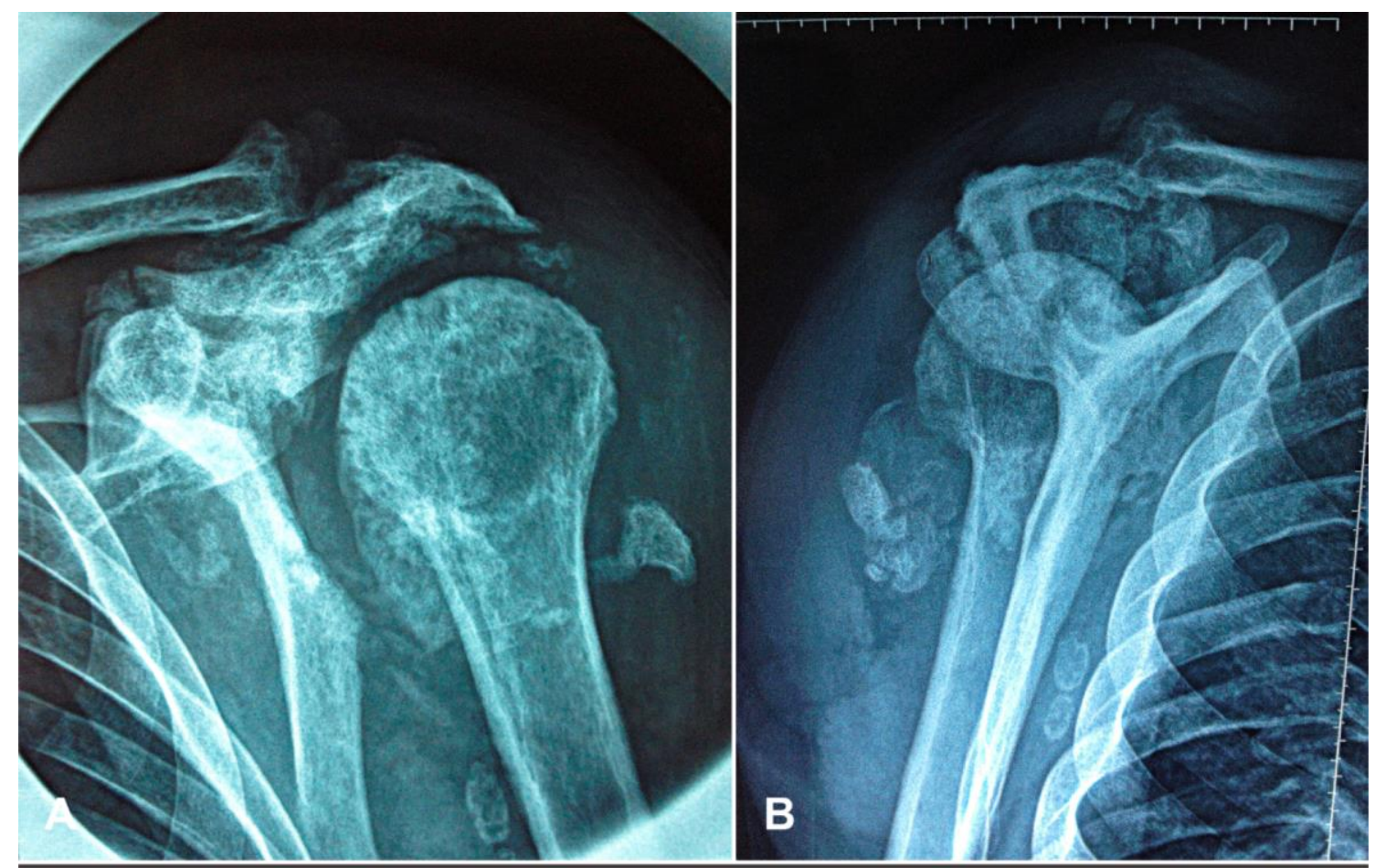

Figura 2 - Radiografia anteroposterior (A) e perfil (B) escapular do ombro esquerdo com sinais de osteoartrose e inúmeros corpos livres osteocondrais periarticulares.

A ressonância magnética (RM) do ombro esquerdo revelou artrose acromioclavicular, osteófitos na cabeça umeral e na glenóide, ruptura completa dos tendões do supraespinal, infraespinal e da maior parte de extensão do subescapular, atrofia dos músculos do manguito rotador e volumoso derrame articular glenoumeral heterogêneo com infiltração de partes moles adjacentes (Figura 3).

O presente relato de caso foi aprovado pelo Comitê de Ética da Associação de Caridade Santa Casa de Rio Grande (CCAE 26093719.8.0000.5303, parecer $\mathrm{n}^{\mathrm{o}}$ 3.811.793). 


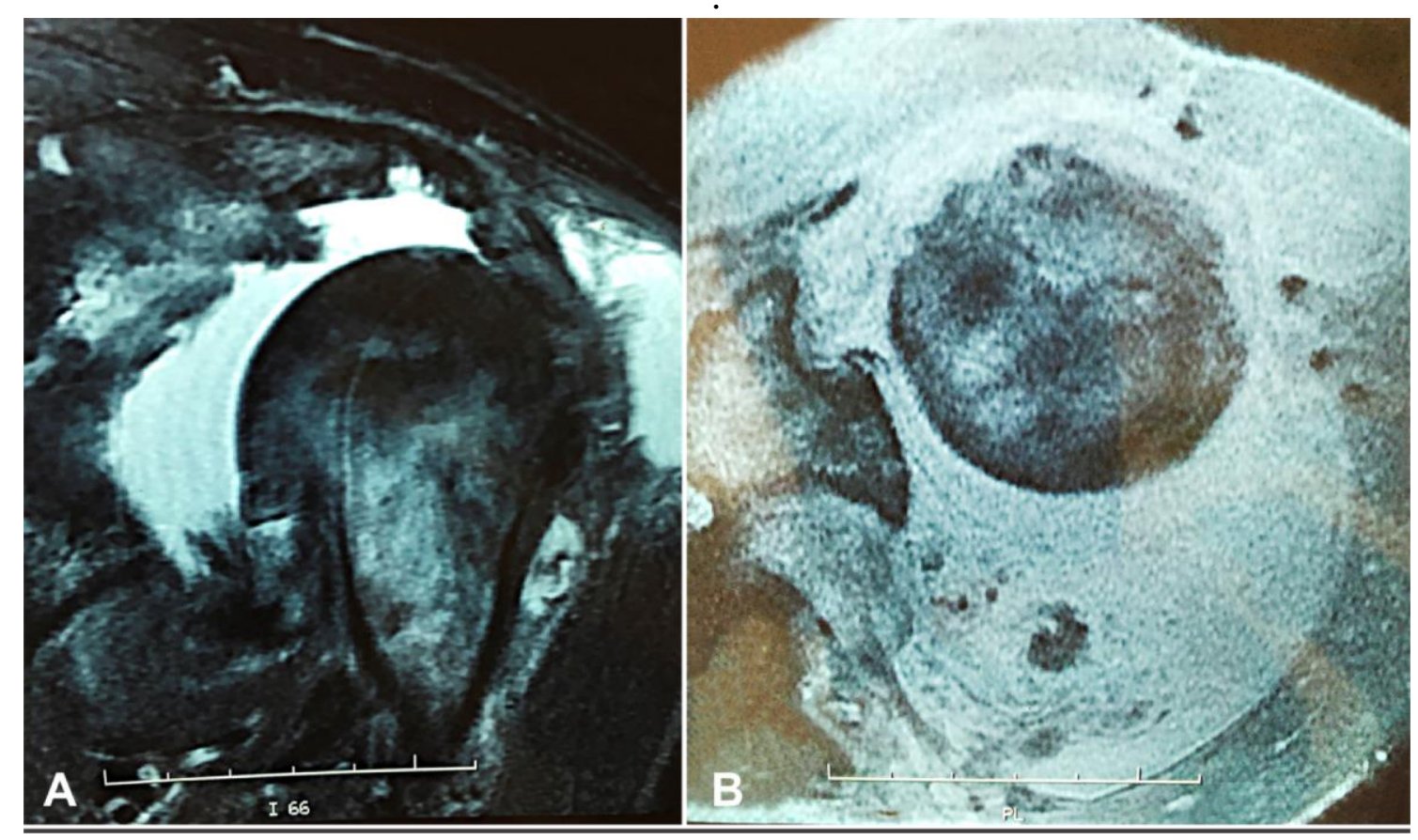

Figura 3 - RM do ombro esquerdo demonstrando grande derrame com intensa destruição da articulação e dos componentes do manguito rotador. (A) Corte Sagital Oblíquo em T2 com Fat Saturation; (B) Corte Axial em T2 com Fat Saturation.

Foi solicitado uma RM da coluna cervical que evidenciou áreas focais de siringomielia nos segmentos cervicais da medula espinhal e espondilodiscoartrose cervical difusa avançada (Figura 4).

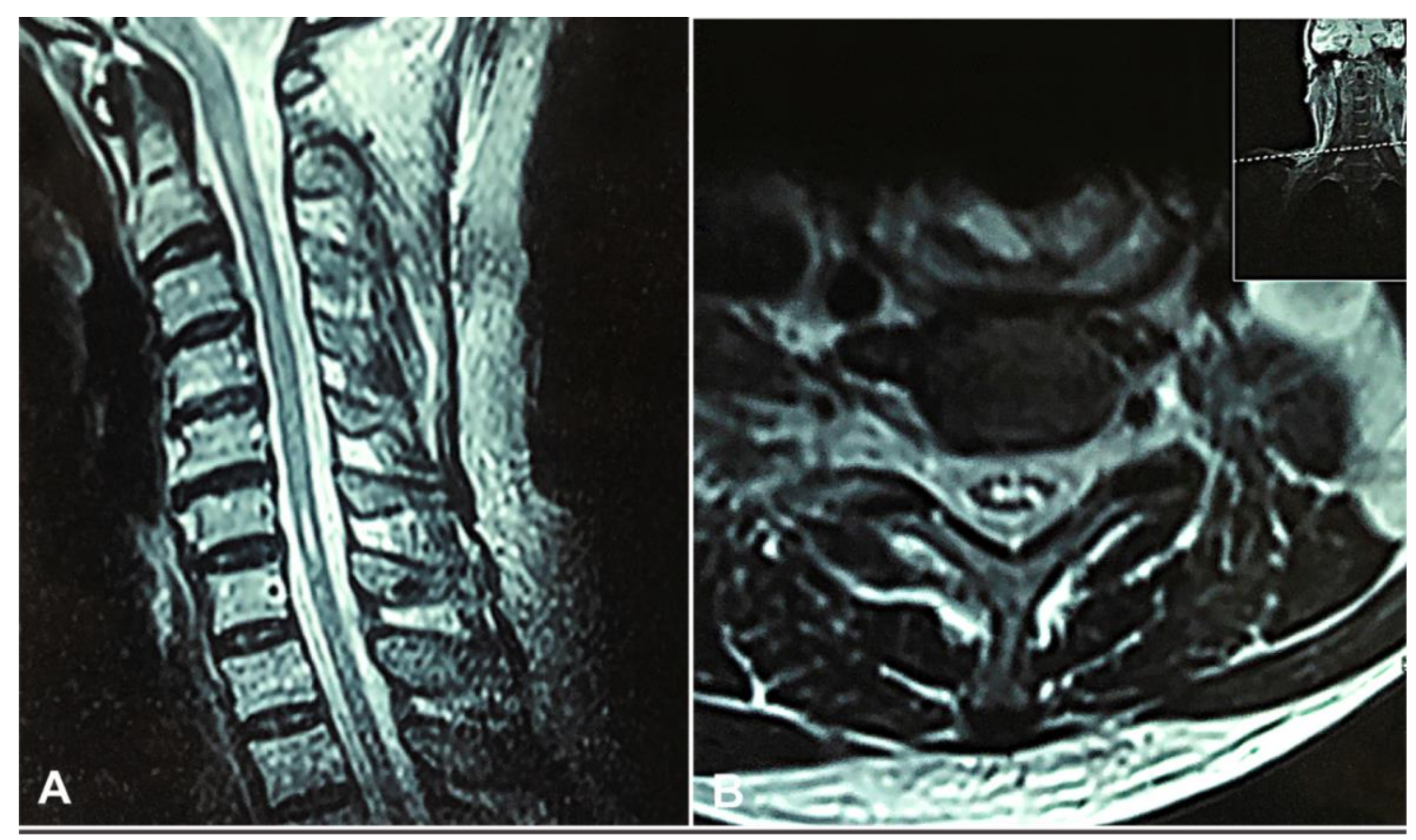

Figura 4 - RM da coluna cervical. (A) Fino traço de hipersinal no centro da medula espinhal no corte sagital em T2 com Fat Saturation à nível cervical. (B) Corte axial constatando dilatação do canal central da medula espinhal. 
O paciente foi submetido ao tratamento conservador com o uso de antiinflamatórios não-esteroidais, órtese e repouso do membro. Atualmente, relata diminuição do derrame articular, manutenção da limitação articular e importante diminuição das queixas álgicas.

\section{Discussão}

A patogênese da NA ainda não está completamente esclarecida, contudo existem duas teorias mais conhecidas: a neurovascular e a neurotraumática $(8,9)$. A teoria neurovascular indica que as alterações articulares resultam da reabsorção óssea ativa exercida pelos osteoclastos, secundária a disfunção simpática que promove angiogênese e hiperemia local persistente (10). Já a teoria neurotraumática propõe que a artropatia é causada por microtraumas de repetição em uma articulação com sensibilidade reduzida. Esta injúria excede a taxa de regeneração tecidual, resultando em lesões macroscópicas como fraturas e luxações que provocam uma deformidade mecânica da articulação. Acredita-se que a teoria neurovascular exerça o papel inicial na etiopatogenia, seguida da teoria neurotraumática que resulta em luxação, fratura e instabilidade $(9,10)$. Dessa forma, é provável que uma combinação destes dois processos seja responsável pelo desenvolvimento da doença (11).

A artropatia neuropática deve ser considerada em casos de inchaço articular inexplicável, desproporcional à dor, com uma amplitude limitada de movimentos ativos e passivos da articulação afetada (9). Em uma análise sistemática de 32 relatos de casos publicados entre 1924 e 2016, a idade média do paciente foi de 49 ( \pm 11 anos). Houve uma prevalência maior em homens (63\%) em comparação com mulheres (37\%). Os sintomas presentes incluíram amplitude de movimento reduzida (90\%), parestesia ou hipoestesia $(76 \%)$, inchaço $(75 \%)$, fraqueza $(68 \%)$, dor $(53 \%)$ e redução dos reflexos tendinosos profundos (37\%), semelhante ao caso apresentado (12).

Em síntese, as manifestações são reunidas na mnemônica dos "6Ds": distensão articular, aumento da densidade, presença de "debris", destruição da superfície articular, deslocamento e desorganização da articulação (8). Caso o ombro seja a articulação afetada, a hipótese de siringomielia precisa ser considerada, visto que a cada quatro pacientes com siringomielia, um irá apresentar NA envolvendo o ombro.

Considerada um distúrbio degenerativo progressivo da medula espinhal, a siringomielia acomete a sensibilidade dolorosa e térmica, mantendo a função motora e a propriocepção. Tais características foram percebidas em nosso paciente. Ela é comumente associada à malformação tipo I de Arnold-Chiari em 75-85\% dos casos. Essa malformação é causada pela migração do tentório do cerebelo inferiormente ao forame magno, aumentando assim o quarto ventrículo. (13). A patogênese da malformação de Chiari associada à siringomielia ainda é um assunto de muito debate na literatura (14). No caso apresentado, não houve associação com a malformação tipo I de Arnold-Chiari.

Dessa forma, estima-se que um terço dos casos de NA ocorra sem diagnóstico de uma etiologia primária (8). Diferenciar a artropatia neuropática de outras artropatias comuns, mesmo que seja um desafio, permanece uma etapa essencial para prevenir a progressão da destruição articular, uma vez que o tratamento se baseia no diagnóstico precoce e no manejo da doença neurológica primária. Em nosso paciente, podemos perceber $o$ diagnóstico tardio, com completa destruição articular que culminou na sua inatividade laboral. A evolução da doença pode ser insidiosa com o paciente por anos assintomático ou com poucos sintomas. Entretanto, o padrão de progressão pode mudar rapidamente e 
gerar uma importante destruição articular, como no caso relatado (11). A RM da coluna vertebral é o padrão ouro para a identificação da siringe e, consequentemente, para o diagnóstico de siringomielia (8).

O tratamento para uma articulação neuropática é inicialmente sintomático. Devem ser feitos encaminhamentos apropriados para abordar qualquer processo patológico identificado como causa da artropatia. A terapia tem o objetivo de retardar a progressão do processo de doença subjacente e preservar ao máximo a funcionalidade da articulação. Por esse motivo, a imobilização e a proteção articular são terapias cruciais para o tratamento das articulações de Charcot (12). Medicamentos antiinflamatórios não-esteróides (AINEs) podem ajudar atenuando a inflamação sinovial e os bifosfonados e a calcitonina podem colaborar inibindo a atividade osteoclástica. A fisioterapia, exercícios de alongamento passivo e órtese funcional ainda são as terapias utilizadas no manejo dessa doença $(8,12,15)$, sendo esse o tratamento de escolha para o caso descrito.

Contudo, nesse estudo retrospectivo, o tratamento foi classificado como tratamento exclusivamente não-cirúrgico (24\%), tratamento operatório $(22 \%)$, terapia combinada (34\%) e nenhum tratamento listado (17\%). A artroplastia foi considerada uma opção viável no controle da dor e melhora do movimento para pacientes com artropatia neuropática após falha do tratamento conservador. No entanto, é um estudo sem acompanhamento a longo prazo, logo não há informação sobre falhas dos componentes e complicações protéticas. (12).

As opções de tratamento cirúrgico para a artropatia de Charcot avançada consistem em desbridamento, artrodese e artroplastias. Embora o alívio da dor a curto prazo seja obtido pelo desbridamento, os efeitos a longo prazo são obscuros. A artrodese limita a função do ombro e tem uma taxa elevada de pseudoartrose, tendo em vista a má qualidade óssea e a idade avançada dos pacientes quando feito o diagnóstico e submetidos ao procedimento (15). A artroplastia é por vezes contraindicada devido aos altos riscos de luxação, afrouxamento, falha do implante e infecção, pois a fraqueza muscular e os distúrbios neurológicos incapacitam o seu funcionamento adequado (15). Por isso, artroplastia ou artrodese é contraindicada na maioria dos casos pelas altas taxas de insucesso. $(13,16,17,18)$. Neste caso, adotamos o tratamento conservador conforme a literatura sugere, porém, por tratar-se de um único caso, afirmar ser a melhor opção é temeroso.

\section{Conclusão}

O neuroartropatia de Charcot no ombro é incomum e a sua associação com siringomielia a torna ainda mais rara, principalmente quando esta não está acompanhada da Síndrome de Arnold-Chiari tipo I. A dificuldade de reconhecer a doença e realizar o diagnóstico diferencial, dado as inúmeras patologias que cursam com sintomas semelhantes, eleva o número de casos de NA sem definição etiológica. Por isso, o cirurgião ortopedista deve estar atento a esses sinais clínicos e radiológicos a fim de evitar falhas diagnósticas e não indicar procedimentos artroplásticos, cada vez mais frequentes em nosso meio, para pacientes onde estes estão contraindicados por suas elevadas taxas de complicações, pelo menos até o momento. Contudo, é importante mencionar a limitação do estudo, visto que não se pode generalizar para todos os casos de NA por se tratar de um caso único amparado a uma literatura escassa já publicada. Conclui-se que são necessários novos estudos, fundamentados em casos com melhores descrições e acompanhamentos a longo prazo para elucidar o melhor tratamento para essa rara enfermidade. 


\section{Conflitos de interesse}

Os autores declaram não haver conflitos de interesse.

\section{Referências}

1. Wang X, Li Y, Gao J, Wang T, Li Z. Charcot arthropathy of the shoulder joint as a presenting feature of basilar impression with syringomyelia: A case report and literature review. Medicine Baltimore 2018; 97(28): 11391.

2. Alai A, Reddy CG, Amrami KK, Spinner RJ. Charcot arthropathy of the shoulder associated with typical and atypical findings. Clinical Anatomy 2012; 26(8): 1017-1023.

3. Kim DH. Charcot Shoulder in a Patient With Tetraplegia. PM\&R 2016; 8(1): 82-84.

4. Vilaça CO, Nascimento OJM, Fiorelli R, Orsini M. Neuroartropatia de Charcot secundária à siringomielia: relato de caso. Rev Bras Neurol 2016; 52(1):18-20.

5. Mazlout O, Larnaout A, El Mufti T, Kammoun N, Ladeb MF. Shoulder arthropathy (Charcot joint) and syringomyelia: Report of two patients. Eur J Orthop Surg Traumatol 2005; 15:37-4.

6. Hatzis N, Kaar TK, Wirth MA, Toro F, Rockwood Jr CA. Neuropathic arthropathy of the shoulder, Journal of Bone and Joint Surgery 1998; 80(9): 1314-1319.

7. Meyer GA, Stein J, Poppel MH. Rapid osseous changes in syringomyelia. Radiology 1957; 69(3):415-8.

8. Sharma A, Panagariya A. Bilateral Charcot arthropathy of shoulder secondary to syringomyelia: an unusual case report. Ann Indian Acad Neurol 2012;1 5(3):2 02-4.

9. Gafoor PMA, Gangadharan P, Mahesh B, Ajith TA. Charcot's osteoarthropathy of the shoulder. International Journal of Orthopaedics Sciences 2016; 2(2): 28-30.

10. Brower AC, Allman RM. The neuropathic joint: a neurovascular bone disorder. Radiologic Clinics of North America 1981; 19(4): 571-580.

11.Memarpour R, Tashtoush B, Issac L, Gonzalez-Ibarra F. Syringomyelia with Chiari I Malformation Presenting as Hip Charcot Arthropathy: A Case Report and Literature Review. Case Reports in Neurological Medicine 2015; 1-6.

12. Rickert MM, Canhão JG, Kirkpatrick JS. Neuropathic Arthropathy of the Shoulder - A Systematic Review of Classifications and Treatments. JBJS reviews, 2019; 7(10): e1.

13. Gaskins III RB, Miller BJ, Scarborough MT. Charcot arthropathy of shoulder: a case report. Orthopaedic Surgery 2011; 3(4): 268-270.

14. Liu H, Wang Y, Yang Z, Wang K. A case report of Charcot arthropathy caused by syringomyelia and Chiari malformation complicated with scoliosis. BMC Research Notes 2014; 7(1): 277.

15. Choudhury P, Mohapatra A, Kharat A, Chauhan V. Neuropathic arthropathy of the shoulder joint caused by syringomyelia: report of a rare case. BMJ Case Reports 2019; 12 (3): e229494.

16. Makihara T, Onishi S, Wadano Y, Fujii K, Nagata H, Sakane M. Regrowth of the deteriorated glenoid in advanced Charcot shoulder after suboccipital decompression for syringomyelia: a case report. Journal of Shoulder and Elbow Surgery 2015; 24(8): 223-228.

17. Yanik B, Tuncer S, Seçkin B. Neuropathic arthropathy caused by Arnold-Chiari malformation with syringomyelia. Rheumatol Int 2004; 24(4): 238-241.

18. Ozkan K, Yavuz U, Akman B, Ozkan NK, Eralp L. Charcot joint of shoulder. Eur J Orthop Surg Traumatol. 2008; 18(3): 229-31.

\section{EDITORA E GRÁFICA DA FURG CAMPUS CARREIROS \\ CEP 96203900 \\ editora@furg.br}

